\title{
A Comparative Study between Thoracic Epidural Anesthesia and General Anesthesia for Patients Who Underwent Modified Radical Mastectomy with Axillary Lymph Node Dissection in De La Salle University Medical Center
}

\author{
Eva Oktavia ${ }^{1, *}$ \\ ${ }^{1}$ Faculty of Medicine, Krida Wacana Christian University, Jl. Arjuna Utara No. 6, Jakarta, Indonesia \\ *Corresponding author. E-mail: eoktaviamd@gmail.com \\ Received date: Jan 19, 2015; Revised date: Mar 18, 2015; Accepted date: Apr 10, 2015
}

\section{Abstract}

$\mathrm{B}$

ACKGROUND: To compare the recovery time and other related clinical outcomes among patients who underwent Modified Radical Mastectomy (MRM) with axillary lymph node dissection under Continuous Thoracic Epidural Anesthesia (CTEA) and General Endotracheal Tube Anesthesia (GETA).

METHODS: A retrospective cross-sectional study with 70 patients who underwent MRM in De La Salle University Medical Centre (DLSUMC), categorized into GETA and CTEA group consisted of 35 patients each. Per oral premedications $15 \mathrm{mg}$ midazolam, $40 \mathrm{mg}$ omeprazole and $10 \mathrm{mg}$ metoclopramide were given 1 hour prior to surgery. Intra-operative hypotension/hypertension, tachycardia/ bradycardia status, length of Post-Anesthesia Care Unit (PACU) and hospital stay, and Post Operative Nausa and Vomiting (PONV) incidence were compared between 2 groups.

RESULTS: Preoperatively, there were no significant differences between the groups in terms of subject characteristic. Intra-operatively, hypertension was more frequent in GETA group (28.6\% vs. $0 \%$ ), while hypotension was more frequent in the CTEA ( $80 \%$ vs. $57.1 \%)$. Tachycardia was more frequent in GETA group $(46.6 \% v s$. $0 \%$ ), meanwhile bradycardia was more frequent in CTEA (40\% vs. 17.1\%). Postoperatively, the GETA group had shorter PACU stay than CTEA (230 mins vs. 267 mins), but CTEA group had a shorter time of hospital stay compared to GETA (58.1 hours vs. 67.7 hours). The incidence of PONV were comparable among the two groups (GETA $46.7 \%$ vs. CTEA $50 \%$ ). Statistically there were no significant differences between the two groups in all of the above characteristics.

CONCLUSION: CTEA technique has no effect on inducing hypertension and tachycardia, but hypotension and bradycardia may occur. Although GETA gives shorter PACU duration, CTEA gives shorter hospital stay. This gave impression that CTEA is an effective alternative technique to GETA in patients who underwent MRM with axillary dissection.

KEYWORDS: modified radical mastectomy, general anesthesia, epidural anesthesia

Indones Biomed J. 2015; 7(2): 111-6

\section{Introduction}

Oncologic breast surgeries have been typically performed under general anesthesia for ages. Although they produce the desired state of unconsciousness, clinical studies showed that this technique (with exception of large dose of opioids) do not eliminate the surgical stress response; it may aggravate immunosuppression and may cause undesirable side effects such as nausea and vomiting up to 24 hours after surgery. The complication has been described by patient as being more debilitating than the mastectomy itself.(1-5) 
Thoracic epidural anesthesia is frequently used for aesthetic breast surgeries, but reports of its use for mastectomies with axillary lymph node dissection are very rare. Clinical evidences suggest that epidural anesthesia has been associated with fewer postsurgical recovery complications and shorter hospital stays, but the adequacy of thoracic and axillary blockade during lymph node dissection is still a problem.(1-5)

Although several research were done using Continuous Thoracic Epidural Anesthesia (CTEA) as alternative of General Endotracheal Tube Anesthesia (GETA) technique during Modified Radical Mastectomy (MRM), they were all foreign studies. It is significant to know the clinical outcome among Filipino patients especially since there was no previous local study. Therefore, this study aimed to compare the recovery time and other related clinical outcomes such as intra-operative hemodynamics and PostOperative Nausea and Vomiting (PONV) among MRM patients who underwent GETA and CTEA technique by measuring duration of stay in Post-Anesthesia Care Unit (PACU) and hospital postoperatively.

\section{Methods}

A retrospective cross-sectional study was reviewed in 70 patients for MRM in De La Salle University Medical Centre (DLSUMC) from July 2008 to June 2013. Written informed consent were obtained from patients prior to surgery. A total of 91 patients' charts were gathered, listed and segregated into GETA group and CTEA group. Adult woman with The American Society of Anesthesiologists (ASA) classification I or II and no history of previous chemotherapy were included in this study. Meanwhile, patients who received intervention of combined GETA and CTEA, GETA with total intravenous anesthesia (TIVA) or GETA with combination of postoperative epidural morphine were excluded from the study. The random number tables were used to randomize the samples until 35 subjects were achieved for each group.

Preoperative evaluation was done a day prior to surgery, recorded in anesthesia preoperative sheet and attached in the patient's chart. Premedications consisted of $15 \mathrm{mg}$ midazolam, $40 \mathrm{mg}$ omeprazole and $10 \mathrm{mg}$ metoclopramide per oral was given 1 hour prior to surgery. GETA was induced with intravenous midazolam, nalbuphine, and propofol. Rocuronium was given to facilitate tracheal intubation. Anesthesia was maintained with sevoflurane/ desflurane/isoflurane in $100 \%$ oxygen. Supplementation of fentanyl was given as analgesia whenever necessary. CTEA was performed using sterile technique, with the patient in left lateral decubitus position. The thoracic epidural space was identified by loss of resistance to air technique. Epidural catheter was inserted into the epidural space through an epidural Touhy needle G18 placed in one of the spaces between T6-T8 vertebrae. The patient was then placed in supine position. Twenty $\mathrm{mL}$ of $0.25 \%$ bupivacaine was administered in $5 \mathrm{~mL}$ fractions. After testing the quality of anesthesia (adequate analgesia from the lower border of the clavicle to the inferior costal margin) using pin prick method, the surgery was initiated. Anesthesia was maintained by injecting $5 \mathrm{~mL}$ of $0.25 \%$ bupivacaine every 60 to 90 minutes through the epidural catheter. Titration of local anesthesia (LA) and sedation of $1-3 \mathrm{mg} / \mathrm{kg} / \mathrm{hr}$ propofol infusion was continued during the surgical procedure.

Preoperative, intra-operative, and postoperative data of each patients were obtained from anesthesia records, PACU notes, and chart progress notes. Preoperative data including age, ASA physical status, diagnosis, type of anesthesia, and duration of surgery. Intra-operative data including tachycardia (heart rate $>100 \mathrm{bpm}$ ) or bradycardia (heart rate $<60 \mathrm{bpm}$ ) status, also hypotension (20\% drop in baseline blood pressure) or hypertension ( $20 \%$ increase in baseline blood pressure) status. Postoperative data including incidence of PONV, length of PACU stay and length of hospital stay. $(1,2,3,6)$

Sample size was computed using OpenEpi version 3.0.1. to detect $60 \%$ frequency of outcome factor in the population, based on study by Belzarena with confidence level of $95 \%$. Patients' demographic data such as age, weight, and duration of surgery was analyzed using descriptive analysis with $95 \%$ confidence interval and presented in means $\pm \mathrm{sd}$. The intra-operative hemodynamic results were tabulated, compared and interpreted through cross tabulation method. The length of PACU stay and the length of hospital stay results were tabulated and represented through histogram. The comparisons of both techniques in terms of length of PACU and hospital stay were measured statistically using measurement of central tendency and t-test. All analyses were done with STATISTICA 6.1.

\section{Results}

The data were categorized into two groups, the GETA group and the CTEA group as shown in Table I. The two groups were compared based on age, weight, ASA classification, duration of surgery, intra-operative hemodynamic, PONV, and the length of PACU and hospital stay.

There were no significant differences between the groups in terms of age, weight and ASA physical status 
classification. Overall, the patients were older women, with $67 \%$ had coexisting morbid diseases (hypertension, ischemic heart disease, coronary arterial disease, diabetes, cerebrovascular disease, asthma, bronchitis, thyroid disorders, anxiety disorders and depression). All patients underwent a unilateral MRM with axillary lymph node dissection. There were no failures in the placement of the epidural catheter. The comparison of the duration of surgery showed that CTEA group had 30 minutes longer duration compared to GETA group.

The intra-operative hemodynamic results were tabulated and compared. The rate of hypertension was more frequent in GETA group ( $28.6 \%$ vs. $0 \%$ ), while hypotension was more frequent in the CTEA $(80 \%$ vs. $57.1 \%)$. Tachycardia was more frequent in GETA group $(46.6 \%$ vs. $0 \%$ ). Meanwhile, bradycardia was more frequent in CTEA group (40\% vs. $17.1 \%)$.

In terms of PONV, this study showed that there was no statistically significant difference between the two groups even though most studies stated that general anesthesia caused a greater frequency and severity of nausea and vomiting than regional anesthesia.

The length of PACU and hospital stay were the primary outcome that was measured to evaluate the patient

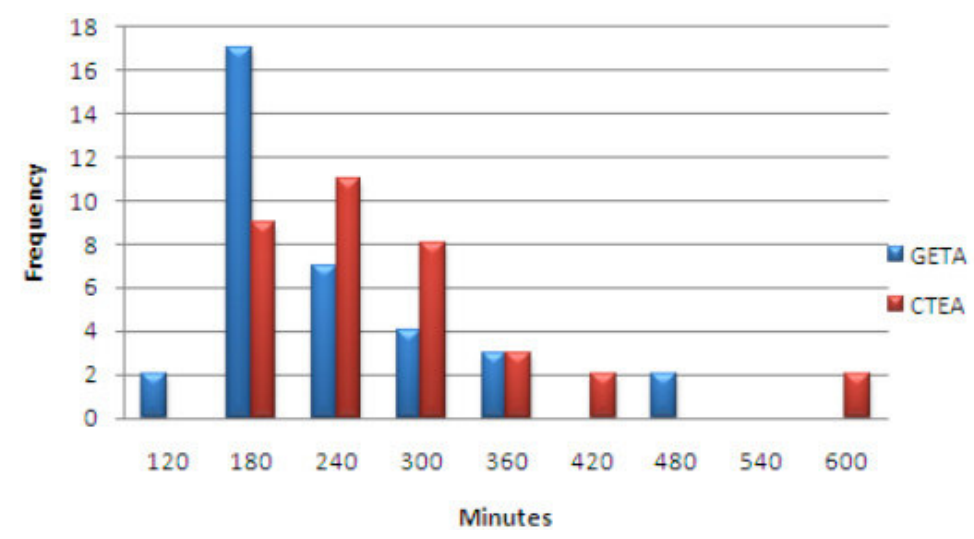

Figure 1. Length of PACU Stay..

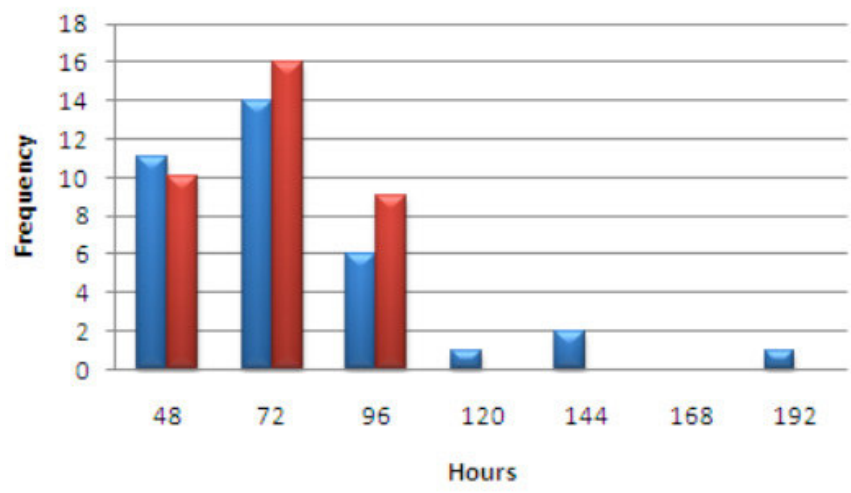

Figure 2. Length of Hospital Stay. recovery time. The result showed that GETA group had 37 minutes shorter PACU stay than CTEA group $(230 \pm 91.2$ minutes vs $267 \pm 104$ minutes) (Figure 1). Although this gave impression that GETA was a better method than CTEA in terms of PACU stay, CTEA group had a shorter time of hospital stay compared to GETA group $(58.1 \pm 12.4$ hours vs $67.6 \pm 30.6$ hours) (Table 1).

In terms of hospital stay most of the patients in CTEA group was discharged after 3 days postoperatively and none of them were hospitalized more than 4 days as compared to GETA group (Figure 2).

Statistical t-test was used to determine the significant of the two groups as regards patient recovery time based on the length of PACU and hospital stay as stated in Table II and Table III. These tables showed that the computed t-value inside the critical value with 0.05 is the level of significance. Therefore, there was no significant difference between the two groups.

\section{Discussion}

Currently, breast cancer is the most common cancer in females and surgical intervention with axillary lymph nodes dissection is still the main treatment. Intervention using radical mastectomy (excision of the breast overlying skin, underlying pectoralis major and the regional lymph nodes) was largely used. Unfortunately, a large number of women still continued to die due to metastasis. Nowadays, randomized trials have demonstrated that intervention using MRM (removal of breast and axillary lymph nodes with the preservation of the pectoralis major muscle) had equivalent patient survival rate to the old radical mastectomy technique. Later, modern therapy evolved into both surgical resection and medical or radiation therapy for treating breast cancer patients. This has allowed dramatic reductions in extensive breast surgery and majority of patients have been now eligible for breast-conversing surgery.(7-9)

Current studies showed that CTEA was a safe and reliable alternative to GETA for women undergoing MRM. The factors which dictated the selection of surgical and anesthetic management were the type and extent of breast cancer, as well as the age, weight, comorbidities, and general condition of the patient.(2) Those preoperative subject characteristics in both groups were statistically comparable in this study.

In clinical practice, it is important to consider 
Table 1. Subject Characteristics.

\begin{tabular}{lcc}
\hline \multicolumn{1}{c}{ Category } & $\begin{array}{c}\text { GETA } \\
(\mathbf{N}=\mathbf{3 5})\end{array}$ & $\begin{array}{c}\text { CTEA } \\
(\mathbf{N}=\mathbf{3 5})\end{array}$ \\
\hline Preoperative Results & & \\
Age (years) & $53.7 \pm 10.7$ & $54.2 \pm 13.7$ \\
Weight (kgs.) & $60.5 \pm 9.82$ & $55.9 \pm 6.17$ \\
ASA & $\mathrm{I}=10(28.6 \%)$ & $\mathrm{I}=13(37.1 \%)$ \\
& $\mathrm{II}=25(71.4 \%)$ & $\mathrm{II}=22(62.9 \%)$ \\
Intra-operative Results & $196 \pm 48.5$ & $226 \pm 52.3$ \\
Duration of Surgery & Hypertension $=10(28.6 \%)$ & Hypertension $=0(0 \%)$ \\
Intra-operative Hemodynamic & Hypotension $=20(57.1 \%)$ & Hypotension $=28(80 \%)$ \\
& Tachycardia $=17(46.6 \%)$ & Tachycardia $=0(0 \%)$ \\
& Bradycardia $=6(17.1 \%)$ & Bradycardia $=14(40 \%)$ \\
Postoperative Results & & $15(50 \%)$ \\
PONV & $14(46.7 \%)$ & \\
\hline
\end{tabular}

Table 2. Length of PACU Stay in Patient Undergoing MRM under Thoracic Epidural Anesthesia and General Anesthesia.

\begin{tabular}{ccc}
\hline Category & $\begin{array}{c}\text { GETA } \\
(\mathbf{N = 3 5})\end{array}$ & $\begin{array}{c}\text { CTEA } \\
\mathbf{N}=\mathbf{3 5}\end{array}$ \\
\hline Mean & 230 & 267 \\
Max & 480 & 570 \\
Min & 120 & 150 \\
Degrees of Freedom & \multicolumn{2}{c}{68} \\
Critical Value & \multicolumn{2}{c}{ \pm 1.995} \\
T & \multicolumn{2}{c}{-1.62} \\
Remark & No significant difference \\
\hline
\end{tabular}

Table 3. Length of Hospital Stay in Patient Undergoing MRM under Thoracic Epidural Anesthesia and General Anesthesia.

\begin{tabular}{ccc}
\hline Category & $\begin{array}{c}\text { GETA } \\
\mathbf{n = 3 5}\end{array}$ & $\begin{array}{c}\text { CTEA } \\
\mathbf{n = 3 5}\end{array}$ \\
\hline Mean & 67.6 & 58.1 \\
Max & 191 & 77 \\
Min & 41 & 43 \\
Degrees of Freedom & \multicolumn{2}{c}{68} \\
Critical Value & \multicolumn{2}{c}{ \pm 1.995} \\
T & \multicolumn{2}{c}{1.7} \\
Remark & No significant difference \\
\hline
\end{tabular}

that either GETA or CTEA technique that will be used should provide adequate intra-operative anesthesia and good postoperative analgesia without collateral effects and with the minimum hospitalization time.(2) As a matter of fact, the quality of anesthesia was adequate in all patients in this study intra-operatively.

When CTEA technique is selected, the problems related with thoracic and axillary innervations. In an axillary dissection, level I and II lymph nodes are removed. These nodes lie behind and lateral to the edge of the pectoralis minor muscle, in this part of surgery the sensory block should reach $\mathrm{C} 4$ dermatome. Thus, when it is tested, as in the present study, the blockade is well established below the clavicle. $(2,9)$ It explains why in this study there was no supplementation of LA infiltration during axillary lymph nodes dissection in CTEA group.

The data of the present study showed that up to $\mathrm{C} 4$ dermatome blockade could be consistently achieved with epidural catheter insertion at the level of low thoracic (T6-T8), threaded $4 \mathrm{~cm}$ cephalad, and administration of $20 \mathrm{~mL} 0.25 \%$ bupivacaine $(\sim 50 \mathrm{mg})$. This technique was supported by a study done

by Visser, et al. They concluded that the total dose of local anesthetic was the most important determinant factor for the extent of the blockade, while the site of epidural puncture controlled the pattern of distribution of sensory blockade. Epidural injection of 10-20 mL LA at lower thoracic segment (T6-L1) would give more cranially sensory blockade (C6T1 to T11-L4) and combination of several patients and technical factors such as age, position, mode and speed of injection may aid in predicting LA dose requirements. As regards epidural catheter insertion, orienting the bevel of the Touhy needle caudal or cranial did not reliably predict final thoracic position related to the puncture site. However, the optional distance to thread a catheter suggested 4-6 cm in epidural space. Threading shorter or longer distances may result in inadequate analgesia or increase incidence of venous cannulation respectively.(10)

Groeben, et al. used $6.6 \mathrm{~mL}$ of $0.75 \%$ bupivacaine ( $49.5 \mathrm{mg}$ ) to perform thoracic block in patients with severe chronic obstructive pulmonary disease (COPD) and asthma. There was no increase in airway obstruction and evoked only mild respiratory motor blocked.(6) Fifty milligrams of bupivacaine that was used in this study was not considered a high dose because MRM procedure did not require intense muscle relaxation and this dose avoided respiratory compromise. All patients in CTEA group were maintained uneventfully with oxygen via face mask at 4-5 lpm perioperatively, even patients with history of respiratory problem. Meanwhile, performing general anesthesia with tracheal intubation in patients with history of coexisting bronchial hyperreactivity could elicit life-threatening bronchospasm.

Hypotension and bradycardia were more frequent 
in CTEA group. This was correlated with the induction of anesthesia after giving loading dose of LA; however, hypotension was easily treated with fluid administration or a single bolus of $10 \mathrm{mg}$ ephedrine if systolic blood pressure decreased below $80 \mathrm{mmHg}$. On the other hand, hypertension and tachycardia was noted in GETA group only. It was correlated with intubation and surgical stimulation especially after skin incision. Aside from increasing the depth of anesthesia, most of the patients in this group received intraoperative increment of boluses of fentanyl with a total of 50-100 $\mu \mathrm{g}$ to decrease the sympathetic response that took place due to surgical stimuli. General anesthesia, although they produce the desired state of unconsciousness, do not eliminate the surgical stress response because of its lack of analgesic properties. Inhalational anesthetics, which was also used in this study, are the most common drugs used for the provision of general anesthesia. Adding a fraction of volatile anesthetic to the inspired oxygen will give a state of unconsciousness and amnesia, but when it is combined with intravenous adjuvants, such as opioids, a balanced technique is achieved that results in analgesia, further hypnosis and amnesia. During operation, large dose of opioid might be needed for maintenance of balance anesthesia in treating adrenergic responses due to noxious stimuli, especially in patients with coexisting cardiovascular disease. Stress associated with anesthesia and surgery results in increased catecholamine levels, increased left ventricular afterload, heart rate, and cardiac complications. Fentanyl is a potent and highly lipid soluble opioid, which has a rapid onset, predictable duration, and high margin of safety for use in clinical anesthesia. Fentanyl can reduce the minimum alveolar concentration (MAC) of volatile anesthetic, and administration of $1.5 \mu \mathrm{g} / \mathrm{kg}$ IV 5 minutes prior to skin incision will reduce the MAC-BAR of volatile anesthetic. $(1,2,5,11,12)$

On the other hand, the benefits of thoracic epidural, which are to selectively block sensory and cardiac sympathetic fibers, attenuate surgical stress response, improve myocardial oxygen balance and stabilize intraoperative hemodynamics, are particularly relevant for patients with coexisting cardiac morbidity.(1)

Regional block has a lower incidence of nausea and vomiting, when compared to general anesthesia, which has been demonstrated in several procedures and studies.(1-6) But in this study, the incidence of this complication was statistically not significant. This might due to the opioids that were used for postoperative pain control in both groups (intravenous oxycodone/tramadol in GETA group and epidural morphine in CTEA group).
Regarding the adjuvants drugs that were used in this study, such as opioids and propofol, nausea and vomiting are among the most distressing side effects of morphine and related opioids. The incidence of opioid-induced nausea and vomiting appears to be similarly irrespective of the route of administration. They induce nausea and vomiting by direct stimulation of the chemoreceptor trigger zone (CTZ) and also by increased vestibular sensitivity such as during postoperative ambulation.(11) In high frequency, it will be distressing to patients and potentially detrimental to their postoperative recovery.

Propofol possess antiemetic properties that can lower the incidence of PONV. In fact, subanesthetic doses of propofol (10 to $20 \mathrm{mg}$ ) have also been successfully used to treat nausea and vomiting in the early postoperative period. $(11,12)$ In this study, even though continuous propofol infusion was done intra-operatively in CTEA group, morphine-induced PONV still prevailed. The possible explanation might be that the antiemetic effects of propofol is more short-lived than the emetic effects of morphine due to accumulation of active metabolite morphine-6glucuronide. In this group, epidural morphine was ordered for 48 hours postoperative.

In this study, the CTEA group had 37 minutes longer PACU stay compared to GETA group. Although it was statistically not significant, some factors that might have influenced this result could be evaluated. Different drugs with different elimination that were used intra-operatively in both groups could affect patient emergence time and would influence the length of PACU stay.

Continued propofol infusion to maintain sedation in CTEA group lasted for an average of 226 minutes surgical duration ( $3-4 \mathrm{hrs})$. The emergence from intravenous hypnosis agents is dependent primarily on its redistribution in body compartments. A $50 \%$ decrease in plasma concentration is required for the awakening at the end of propofol infusion. Thus, duration of unconsciousness is affected by many things, such as context-sensitive halflive, amount of drug, co-administration with other drug (opioids, benzodiazepine) and patient's factors. Desflurane, sevoflurane, and isoflurane are the most popular potent inhaled anesthetics used in adult surgical procedure, and was also used in our GETA group. Inhaled anesthetics allow rapid emergence because of its easy titratibility. Emergence from inhalational anesthesia depends on pulmonary elimination of the drug and MAC awake (the end-tidal concentration associated with eye-opening to verbal command) which is approximately $30 \%$ of MAC (desflurane $2.17 \%$, sevolurane $0.61 \%$, isoflurane $0.39 \%$ ). Time of emergence is quicker 
in gas with lower blood-gas solubility (desflurane $4 \mathrm{~min}$, sevoflurane $7 \mathrm{~min}$, isoflurane $11.5 \mathrm{~min}) .(11-15)$

Another factor that might prolong PACU stay are the incidence of PONV and human factors, such as late discharge order from attending anesthesiologist.

The length of hospital stay in CTEA group was 9.5 hours shorter than GETA group. This is important in measuring patient recovery time since the patient could readily return to their families and social environment. Unfortunately, in a deeper statistical analysis, this time difference showed no statistical significance. The use of opioids for postoperative analgesia in both groups for 24-48 hours might explain the delay of patients' recovery time. Although it gave adequate postoperative pain control in both groups, it also caused prominent nausea and vomiting that prevented the patient to have early ambulation and oral feeding. Study by Doss et al also gave the same result as this study, but instead of epidural morphine they used intravenous hydromorphine or meperidine for postoperative analgesia in both GETA and CTEA groups. $(1,11)$

\section{Conclusion}

Preoperatively, subject characteristics between the two groups in this study were comparable. Intra-operative hemodynamic results suggested that CTEA technique has no effect on inducing hypertension and tachycardia, but hypotension and bradycardia may occur. Although GETA has a shorter duration than CTEA in terms of PACU stay, CTEA had a shorter time of hospital stay compared to GETA. This gave impression that CTEA was a safe and reliable alternative technique to GETA for patient undergoing MRM with axillary dissection. Successful use of CTEA in MRM surgery could avoid problems of difficult tracheal intubation, stress of anesthesia and surgery and hemodynamic changes associated with tracheal intubation. Nevertheless, it is important to always remember that patients' general condition and the extensiveness of surgery should become main consideration as regards the selection of anesthetic management.

\section{References}

1. Doss NW, Ipe J, Crimi T, Raipal S, Cohen S, Fogler RJ, et al. Continuous thoracic epidural anesthesia with $0.2 \%$ ropivacaine versus general anesthesia for perioperative management of modified radical mastectomy. Anesth Analg. 2001; 92: 1552-7.

2. Belzarena SD. Comparative study between thoracic epidural block and general anesthesia for oncologic mastectomy. Rev Bras Anesthesiol. 2008; 58: 561-8.

3. Lynch EP, Welch KJ, Carabuena JM, Eberlein TJ. Thoracic epidural anesthesia improves outcome after breast surgery. Ann Surg. 1995; 222: 663-9.

4. Vookles J. Consider epidural anesthetic as an adjunct or primary technique for patients having mastectomy. In: Marcucci C, Cohen NA, Metro DG, Kirsch JR, Marcucci L, editors. Avoiding common anesthesia errors. Philadelphia: Lippincott Williams and Wilkins; 2008. p. 456-7.

5. Hiremath VR. Thoracic epidural anesthesia for modified radical mastectomy-in type2 diabetes mellitus patient. J of Evolution of Med and Dent Sci. 2014; 3: 15002-6.

6. Groeben H, Schafer B, Pavlakovic G, Silvanus MT, Peters J. Lung function under high thoracic segmental epidural anesthesia with ropivacaine or bupivacaine in patients with severe obstructive pulmonary disease undergoing breast surgery. Anesthesiology. 2002; 96: 536-41.

7. Acosta J, Alacron LH, Anaya DA, Ashley SW, Auerbach PS, Azizzadeh A, et al. Townsend: sabiston text book of surgery. 18th ed. USA: Elsevier Inc; 2008.

8. Brunicardi FC, Andersen DK, Billiar TR, Dunn DL, Hunter JG, Pollock RE. Schwartz's principles of surgery. 8th ed. New York: McGraw-Hill; 2006.

9. Jaffe RA, Samuels SI, Schmiesing CA, Golianu B. Anesthesiologist's manual of surgical procedures. 4th ed. Philadelphia: Lippincott Williams and Wilkins; 2009.

10. Visser WA, Lee RA, Gielen MJM. Factors affecting the distribution of neural blockade by local anesthetics in epidural anesthesia and a comparison of lumbar versus thoracic epidural anesthesia. Anesth Analg. 2008; 107: 708-21.

11. Barash PG, Cullen BF, Stoelting RK, Cahalan MK, Stock MC. Handbook of Clinical Anesthesia. 6th ed. Philadelphia: Lippincott Williams and Wilkins; 2009.

12. Stoelting RK, Hillier SC. Pharmacology and physiology in anesthetic practice. 4th ed. Philadelphia: Lippincott Williams and Wilkins; 2006.

13. Sinclair RCF, Faleiro RJ. Delayed recovery of consciousness after anesthesia. BJ Anesth. 2006; 6: 114-8.

14. Hill SA. Pharmacokinetics of drug infusions. BJ Anesth. 2004; 4: 7680.

15. Jindal R, Kumra VP, Narani KK, Sood J. Comparison of maintenance and emergence characteristics after desflurane or sevoflurane in outpatient anesthesia. Indian J Anaesth. 2001; 55: 36-42. 